\title{
ASYMMETRIES IN THE SPECTRAL LINES OF EVOLVED HALO STARS*
}

\author{
David F. Gray ${ }^{1}$, Bruce W. CARneY ${ }^{2}$, AND David Yong ${ }^{3}$ \\ ${ }^{1}$ Department of Physics \& Astronomy, University of Western Ontario, London, ON N6A 3K7, Canada; dfgray @ uwo.ca \\ ${ }^{2}$ Department of Physics \& Astronomy, University of North Carolina, Chapel Hill, NC 27599-3255, USA; bruce@ unc.edu \\ ${ }^{3}$ Mount Stromlo Observatory, Research School of Astronomy and Astrophysics, Australian National University, Cotter Road, Weston Creek, ACT 2611, Australia; \\ yong@mso.anu.edu.au \\ Received 2007 December 15; accepted 2008 March 10; published 2008 April 24
}

\begin{abstract}
We do an initial reconnaissance of asymmetries of spectral lines in metal-poor field stars using high-resolution observations of four red horizontal-branch and 11 red giant branch stars taken with the coude spectrograph at the Canada-France-Hawaii Telescope. We find that (1) the shapes of the line bisectors for metal-poor stars hotter than $4100 \mathrm{~K}$ mimic the well-known $\mathrm{C}$ shape of bisectors for solar-metallicity stars on the cool side of the granulation boundary while (2) metal-poor stars cooler than $4100 \mathrm{~K}$, or higher up the red giant branch than $M_{\mathrm{V}}$ $=-1.5$, show bisectors with a reversed-C shape, similar to those for solar-metallicity stars on the hot side of the granulation boundary and similar to the reversed-C shape found in a previous study for the M-type supergiant Betelgeuse. The well-documented radial-velocity jitter of high-luminosity stars and the line bisector characteristics vary in concert up the red giant branch; both phenomena are probably signatures of large convection cells.
\end{abstract}

Key words: convection - stars: oscillations - stars: variables: other - supergiants - techniques: spectroscopic

Online-only material: color figures

\section{THE CONTEXT}

In a bid to understand the line broadening of halo stars, Carney et al. (2008a) obtained high-resolution spectra, $\lambda / \Delta \lambda \sim 120,000$, with a moderately high signal-to-noise ratio $(\mathrm{S} / \mathrm{N}), \sim 200$, for a sample of seven red horizontal-branch stars and 12 red giant branch stars to which they applied a full Fourier analysis for rotation and macroturbulence. The contribution to line broadening by macroturbulence was found to be strong and similar to Population I stars. Rotation was measurable, although for the highest luminosity stars $\left(M_{\mathrm{V}}<1.5\right)$, the apparent projected rotation rates, $v$ sin $i$, were a small fraction of the broadening and therefore might actually arise from other mechanisms, possibly pulsation or shock waves, for example. In earlier publications and with much larger numbers of stars, radial-velocity jitter, i.e., noise-like fluctuations up to several $\mathrm{km} \mathrm{s} \mathrm{s}^{-1}$, was documented but not satisfactorily explained (Carney et al. 2003, 2008b).

One of the limitations of the halo-star data in Carney et al. (2008a) is the sparse-to-non-existent time coverage. These stars are faint and limited telescope time was available, so often only one exposure was taken. In a completely independent study (Gray 2008), line profiles from hundreds of exposures of the Population I supergiant Betelgeuse (HR 2061, M2 Iab, $\left.M_{\mathrm{V}} \sim-5\right)$ were analyzed. The radial velocity variations of Betelgeuse have amplitudes and time variations similar to the jitter observed in many stars near the top of the red giant branch. The collective Betelgeuse evidence strongly suggests that the motions of large convection cells and oscillations they induce is the cause of radial-velocity jitter.

Another characteristic of Betelgeuse is the predominance of the reversed-C shape of the spectral-line bisectors. (The bisectors are variable.) Asymmetries impressed on spectral lines by Doppler-shift distributions in stellar photospheres are well

* Based on observations obtained at the Canada-France-Hawaii Telescope (CFHT) which is operated by the National Research Council of Canada, the Institut National des Sciences de l'Univers of the Centre National de la Recherche Scientifique de France, and the University of Hawaii. documented for the Sun and other Population I stars (e.g., Beckers 1981; Livingston 1982; Dravins et al. 1981; Brandt \& Solanki 1990; Gray 1980, 1982, 2005a). The asymmetries are generally specified using the bisector of the spectral line, and the observed bisectors fall into two broad morphological categories: $\mathrm{C}$ shaped and reversed-C shaped. In earlier investigations, the two types were found to be segregated in the H-R diagram. Those with the $\mathrm{C}$ shape are on the cool side of the granulation boundary that runs almost vertically from about F0 on the main sequence to G0 for supergiants (Gray \& Nagel 1989; Gray 2005a) and includes the Sun, while those with reversed-C shapes lie on the hot side of the boundary.

The C shape of the bisectors of cool stars actually shows considerable variation with effective temperature and luminosity (Gray 2005a, 2005b). It is generally accepted that the main process producing the $\mathrm{C}$ shape is the Doppler-shift distribution of granulation, which in turn depends on the strength of the convective velocity fields, the amount of overshoot through the photosphere, and the brightness contrast between hot rising material and cool falling material. Other velocity fields, such as non-radial oscillations, contribute to the broadening of spectral lines, but probably not to their asymmetries. Hydrodynamical models have been remarkably successful in reproducing the observed asymmetries in solar lines (Asplund et al. 2000). On the other hand, the physical cause of reversed-C-shaped bisectors is not known and very few studies have been made.

The reversed-C shape for Betelgeuse opens another window into velocity fields, being the first case to be found on the cool side of the granulation boundary. It is therefore natural to enquire if this is characteristic of all red giant branch stars. Further, if this is a signature of large convection cells, lengthy series of observations may not be needed to identify the process and deduce some of its characteristics. The reversed-C-shaped bisector may be sufficient. We investigate here the subset of the exposures used by Carney et al. (2008a) that have high enough $\mathrm{S} / \mathrm{Ns}$ to allow bisectors to be measured. This paper documents our findings. 

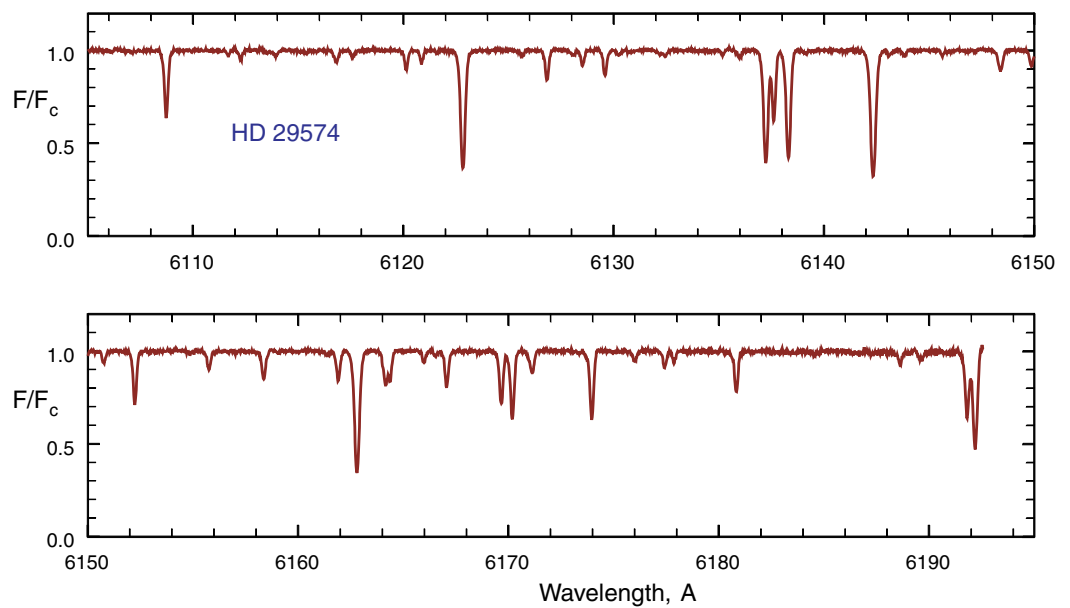

Figure 1. This exposure of HD 29574 illustrates the quality of the observations and the relatively few number of lines suitable for measuring bisectors. The ordinate $F / F_{\mathrm{c}}$ is flux normalized to the continuum.

(A color version of this figure is available in the online journal)

Table 1

Exposure and Star Information

\begin{tabular}{lccccccccc}
\hline \hline Star & $T_{\text {eff }}$ & $\log \mathrm{g}$ & {$[\mathrm{Fe} / \mathrm{H}]$} & Type $^{\mathrm{a}}$ & $\lambda$ & Julian date $^{\mathrm{b}}$ & $\mathrm{S} / \mathrm{N}$ & $\mathrm{N}^{\mathrm{c}}$ & Bi $^{\mathrm{d}}$ \\
\hline HD 3008 & 4140 & 1.00 & -1.43 & RGB & 6150 & 3366.7716 & 175 & 6 & ( \\
CD - 36 1052 & 5890 & 2.50 & -2.00 & RHB & 5430 & 3366.8083 & 150 & 2 & ( \\
HD 23798 & 4310 & 1.00 & -1.90 & RGB & 6150 & 3366.8681 & 210 & 4 & ( \\
HD 25532 & 5320 & 2.54 & -1.33 & RHB & 5430 & 4016.9720 & 175 & 5 & ( \\
BD +6 648 & 4160 & 0.87 & -1.82 & RGB & 6150 & 4015.1177 & 285 & 4 & ) \\
HD 29574 & 3960 & 0.57 & -2.11 & RGB & 6150 & 3366.8934 & 155 & 7 & ) \\
HD 82590 & 5960 & 2.70 & -1.85 & RHB & 6150 & 3366.9454 & 180 & 3 & ( \\
BD +22 2411 & 4320 & 1.00 & -1.95 & RGB & 6150 & 3367.0135 & 160 & 6 & ( \\
HD 110281 & 3850 & 0.2 & -1.75 & RGB & 6150 & 3367.0801 & 170 & 9 & ( \\
HD 165195 & 4200 & 0.76 & -2.16 & RGB & 6150 & 4011.7779 & 380 & 5 & ( \\
HD 184266 & 5490 & 2.60 & -1.87 & RHB & 5430 & 4016.7436 & 250 & 4 & ( \\
HD 187111 & 4260 & 1.04 & -1.65 & RGB & 6150 & 4015.8079 & 395 & 6 & ( \\
HD 214295 & 3890 & 0.30 & -2.14 & RGB & 6150 & 4015.7595 & 215 & 6 & ) \\
HD 218732 & 3900 & 0.20 & -2.00 & RGB & 6150 & 4015.8478 & 270 & $6 ?$ & ) \\
HD 221170 & 4410 & 1.10 & -2.26 & RGB & 6150 & 4009.8693 & 260 & 7 & ( \\
\hline
\end{tabular}

Notes.

${ }^{a}$ RGB denotes red giant branch; RHB denotes red horizontal branch.

b Julian date of mid exposure minus 2450000.

${ }^{\mathrm{c}}$ Number of useable bisectors for this exposure.

d Type of bisector shape.

\section{THE OBSERVATIONS}

The data were taken with the high-resolution Gecko coude spectrograph at the Canada-France-Hawaii telescope. As noted above, the resolving power of this spectrograph is $\sim 120,000$. We used the grating in two orders, observing 5385-5470 in the tenth order and 6105-6190 $\AA$ in the ninth order. The CCD detector was thinned, with 2048 by $409615 \mu$ pixels, corresponding to $\sim 0.022 \AA$ per pixel, and a read-out noise of 7.5 electrons, which is negligible for our exposures. The continuum $\mathrm{S} / \mathrm{N}$, as estimated from the photon count, ranges from 150 to 395 per column.

The selected stars include four on the red horizontal branch and 11 on the red giant branch. Table 1 lists the stars and related information. Figure 1 shows a sample spectrum. The number of usable spectral lines varies from 2 to 9 , depending on the star. Owing to the low metallicity of these stars, only a few lines are deep enough to yield bisectors, but on the positive side, the bisector distortions from line blending are minimal.
Additional information concerning these stars and their observations can be seen in Carney et al. (2008a). Although there is a possibility that some of our red giant branch stars are actually on the asymptotic giant branch (AGB), this does not alter their luminosity and surface gravity significantly. Consequently, we presume the physics of the line formation to be unaffected.

\section{EXAMPLES OF BISECTORS FOR POPULATION II STARS}

It is important to remember that we are exploring here the shapes, not the absolute positions, of the bisectors of spectral lines. Arbitrary horizontal shifts for convenience of display are made in the figures. A selection of bisectors with the $C$ shape is shown in Figure 2. Located under the bisectors of each panel is a horizontal error bar typical of each exposure. They were estimated from the $\mathrm{S} / \mathrm{N}$ in the continuum translated through the slope of the sides of the line profiles. The errors grow to infinity at the very center of a line and at the continuum, as the slope goes to zero (see Gray 1983). 

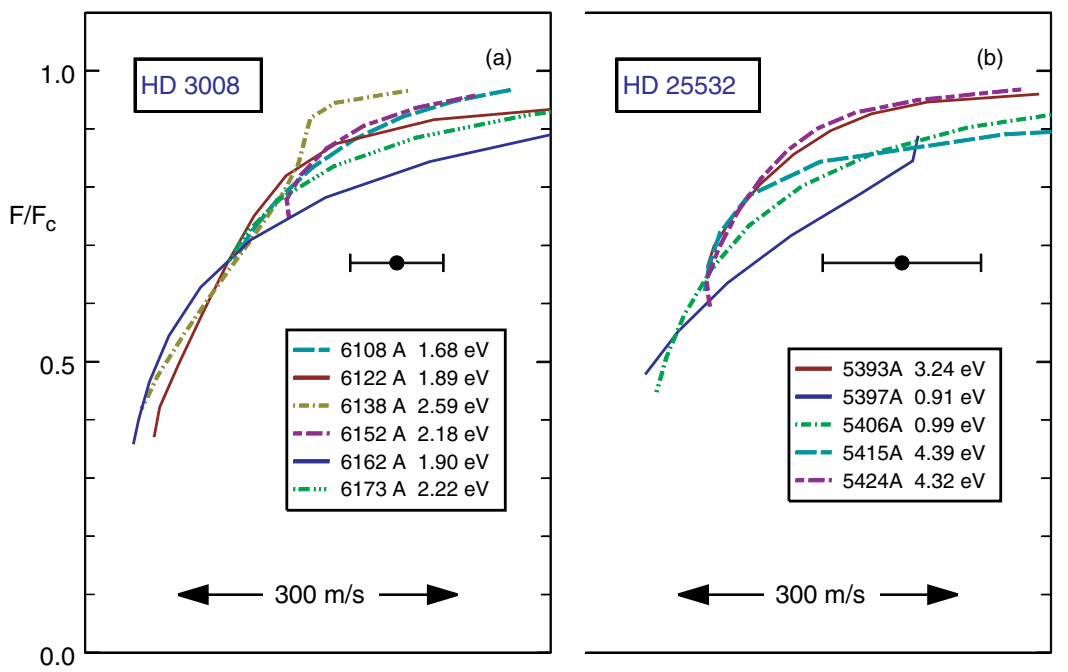

Figure 2. The spectral-line bisectors of these two stars illustrate the top of the C shape. (a) The 6150 A region for HD 3008 . (b) The $5400 \AA$ region for HD 25532. The horizontal error bar shows the typical error on the bisector points.

(A color version of this figure is available in the online journal)

Most of our program stars in this category do not show the bottom portion of the $\mathrm{C}$, and in this regard are similar to the well-documented case of the disk star Procyon (H-R 2943, F5 IV-V, Gray 1982, 2005a; Dravins 1987). An observation of the metal-poor subgiant, HD 140283 with $[\mathrm{Fe} / \mathrm{H}] \sim-2.7$, by Allende Prieto et al. (1999) also shows mainly the top of the C shape. The likely interpretation is that rising granules continue to dominate up to at least those photospheric layers where the line cores are formed. This follows naturally in the sense that lower metallicity means fewer electron donors and so less opacity from the negative hydrogen ion. Essentially, the photosphere is more transparent and we see deeper into the granulation. However, it should also be noted that owing to the low metallicity, few of the lines have deep cores, where the bottom of the $\mathrm{C}$ is found.

In studies of cool disk stars, it is normal to be able to superimpose bisectors with a horizontal shift and no other scaling. An example of this can be seen for Procyon (Gray 2005a). Although our Population II stars are also on the cool side of the granulation boundary and some show a (top of the) $\mathrm{C}$ shape, it is not clear if their bisectors behave in this manner because of the relatively large observational errors. Observations at higher $\mathrm{S} / \mathrm{Ns}$ and with additional spectral lines should resolve this point.

Two of the red horizontal-branch stars have significant rotation, $\mathrm{CD}-36^{\circ} 1052$ with $v \sin i=8.8 \mathrm{~km} \mathrm{~s}^{-1}$ and HD 82590 with $v \sin i=7.7 \mathrm{~km} \mathrm{~s}^{-1}$ (Carney et al. 2008a). As shown in Figure 3, both stars show a full C-shape bisector, and because rotation makes the lines more shallow, these bisectors appear vertically compressed. The redistribution of the Doppler shifts of granulation by those of rotation has been studied previously in simple models (Gray 1985, 1988), but in all those cases, rotation increased the blueward shift of the core portion of the bisectors, opposite to what we see for these two stars. Perhaps the models in these former studies were too simple to apply to red horizontal-branch stars.

An example of bisectors with the reverse-C shape is shown in Figure 4(a). Although these are cool stars, their bisectors look similar to solar-metallicity stars on the hot side of the granulation boundary. Just as with the solar-metallicity stars, each spectral line has its own bisector shape and they cannot be matched

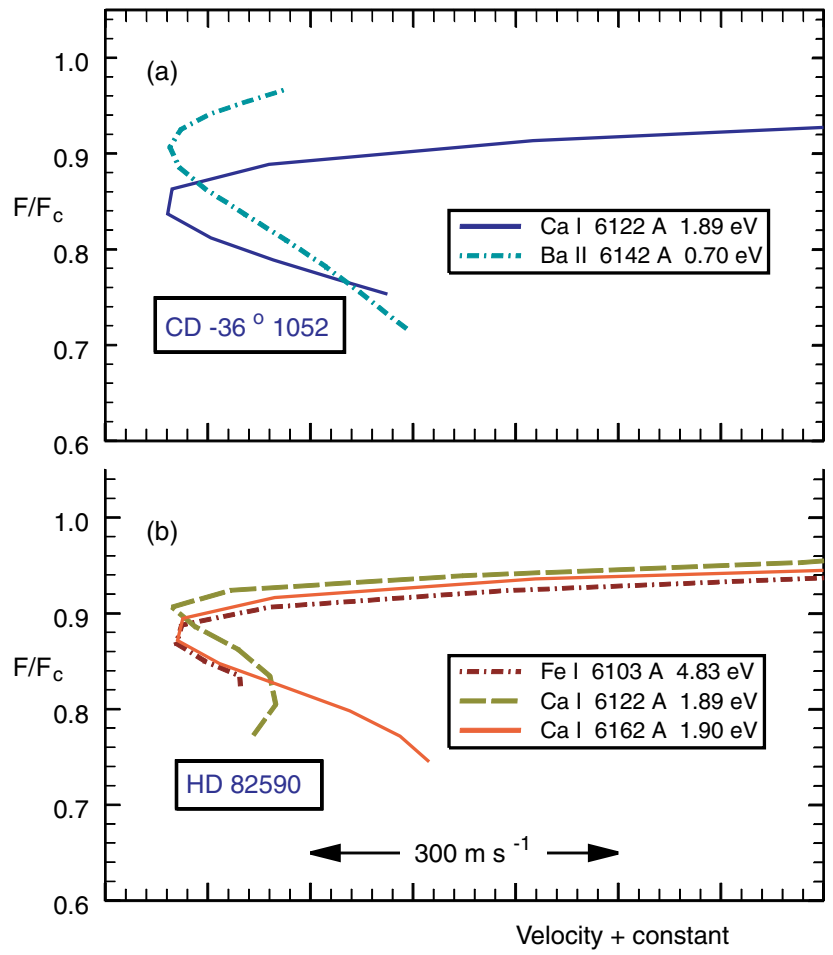

Figure 3. These two stars have more rapid rotation than some of the others in our sample; $v$ sin $i=8.8 \pm 0.8 \mathrm{~km} \mathrm{~s}^{-1}$ for $\mathrm{CD}-36^{\circ} 1052$ and $7.7 \pm$ $0.6 \mathrm{~km} \mathrm{~s}^{-1}$ for HD 82590 . The profiles are therefore more shallow, resulting in vertical compression of the bisectors.

(A color version of this figure is available in the online journal)

by horizontal shifting (Gray 1989). It is possible, however, to rescale the bisectors using an arbitrary re-positioning of the zero level. This is shown in Figure 4(b). The scaling does not result in a perfect match, but it is close, given the $S / N$ s of these data. We have not yet found a physical explanation, if there is one, to the zero-level re-adjustment factors. They are not correlated with the core depths of the lines. 

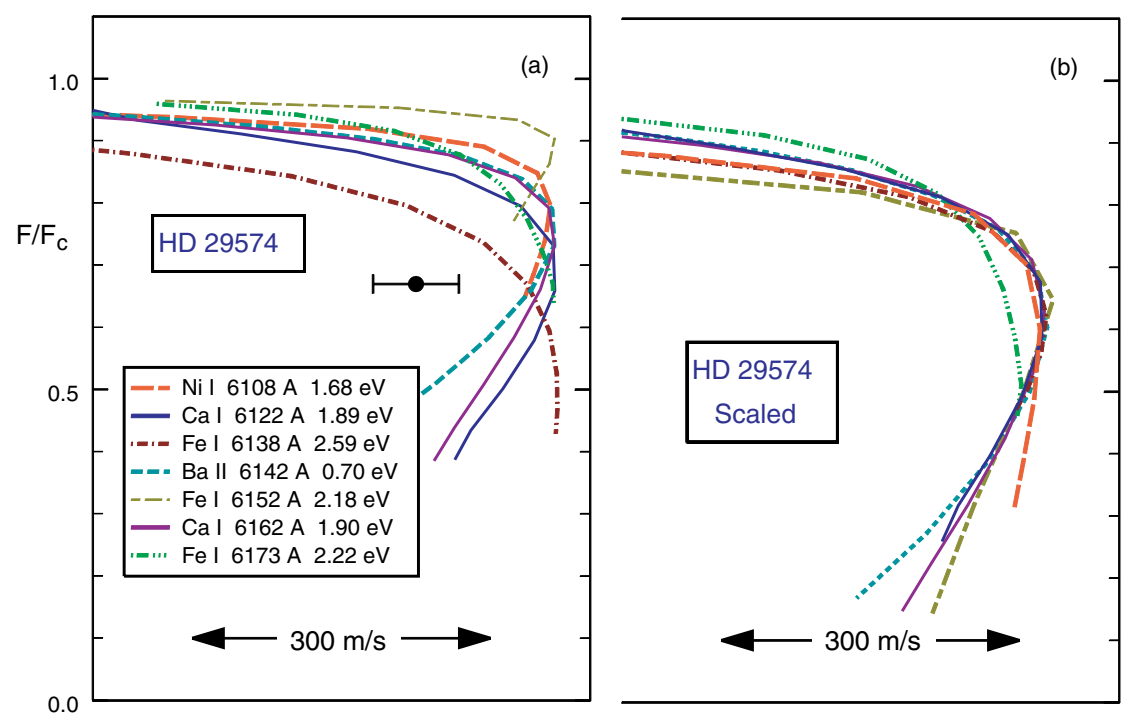

Figure 4. The bisectors of HD 29574 illustrates the reversed-C shape. (a) The bisectors as measured. The horizontal error bar shows the typical error on the bisector points. (b) The same bisectors with arbitrary zero-level scaling.

(A color version of this figure is available in the online journal)

\section{BISECTOR VARIATION WITH POSITION IN THE H-R DIAGRAM}

Shapes of bisectors are indicators of the nature and depth dependence of photospheric velocity fields, and as such serve as basic observational constraints for hydrodynamical models. All of our program stars are at or on the cool side of the granulation boundary, and on that basis C-shaped bisectors would be anticipated. As already noted, this is not the case. As summarized in Figure 5, the red horizontal-branch stars and the red giant branch stars hotter than $4100 \mathrm{~K}$ show C-shaped bisectors, but red giant branch stars cooler than $4100 \mathrm{~K}$ show the reversed-C shape. The segregation is apparently not complete, since there are two exceptions: $\mathrm{BD}+6^{\circ} 648$ shows the reversedC shape and is hotter than $4100 \mathrm{~K}$ and HD 110281 has a C shape and is cooler than $4100 \mathrm{~K}$. The first star may simply be at the boundary between the two cases, given the $\sim 100 \mathrm{~K}$ errors in the effective temperatures. HD 110281 is the coolest star in our sample and the closest to Betelgeuse, although substantially fainter. Betelgeuse is variable and occasionally shows C-shaped bisectors. Possibly HD 110281 is variable, like Betelgeuse, and we observed it at one of those rare times when the bisector is opposite its normal shape.

Those stars known to show velocity jitter according to Carney et al. (2008a, 2008b) are marked in Figure 5. In most cases, the jitter is based on ten or more velocity measurements spanning at least 2900 days. However, we have also placed BD $+6^{\circ} 648$ in this category based on four velocities spanning only 403 days from Carney et al. (2008b) and one velocity from Carney et al. (2008a). In a similar manner, HD 187111 is placed in the non-jitter group based on only five velocities spanning 781 days from Carney et al. (2008b).

Although stars showing jitter occupy the same region of the HR diagram as those having reversed-C bisectors, there appears to be a less than perfect correspondence between the two phenomena. This is curious. To further explore, we estimated the velocity span of the upper portion of the bisectors from $90 \%$ of the continuum to the extreme velocity of the $\mathrm{C}$ or reversed $\mathrm{C}$. The velocity span is chosen to be positive for $\mathrm{C}$

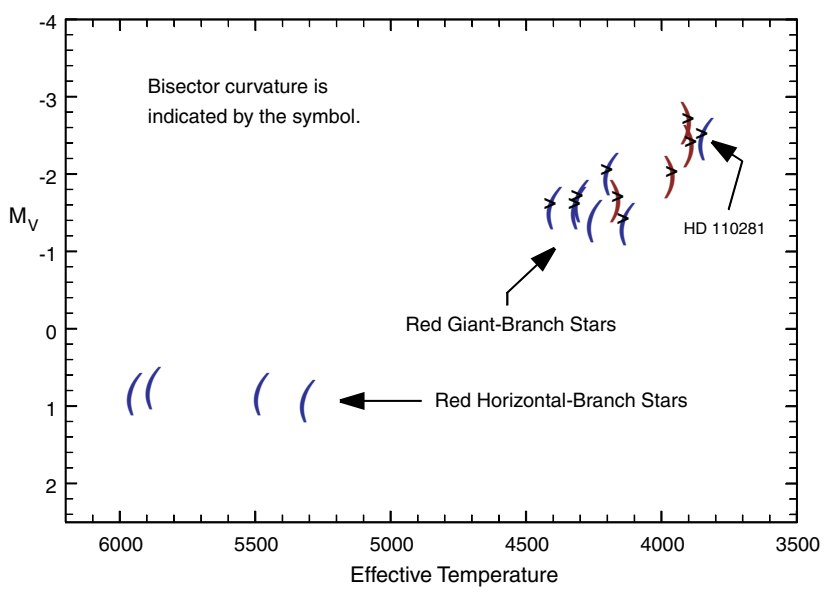

Figure 5. Qualitative bisector shapes are shown as a function of position in this $\mathrm{H}-\mathrm{R}$ diagram. The reversed-C shaped bisectors are in the upper portion of the red giant branch. Stars known to exhibit velocity jitter are marked with a $>$.

(A color version of this figure is available in the online journal)

shapes and negative for reversed-C shapes. The process has a subjective component since different bisectors in the same star have somewhat different velocity spans, so the errors are large. Nevertheless, a systematic change in velocity span with position in the H-R diagram is uncovered, as shown in Figure 6. Also shown on this plot are the measured rms jitter values for those of our stars showing it, taken from Carney et al. (2003, 2008b). Jitter and bisector velocity span track each other up the red giant branch. Although the data are sparse, the transition to reversedC shapes probably does not occur abruptly when a star evolves across $\sim 4100 \mathrm{~K}$. A smooth transition is also consistent with the continuous and monotonic increase in estimated convections' cell dimensions (e.g., Schwarzschild 1975; Freytag et al. 2002).

HD 110281 does not fit this relation and is completely off scale with a velocity span $\sim+700 \mathrm{~m} \mathrm{~s}^{-1}$. This star deserves more study.

To see the effect of metallicity, we compare in Figure 7 the bisectors of our program star HD 3008 with three stars having 


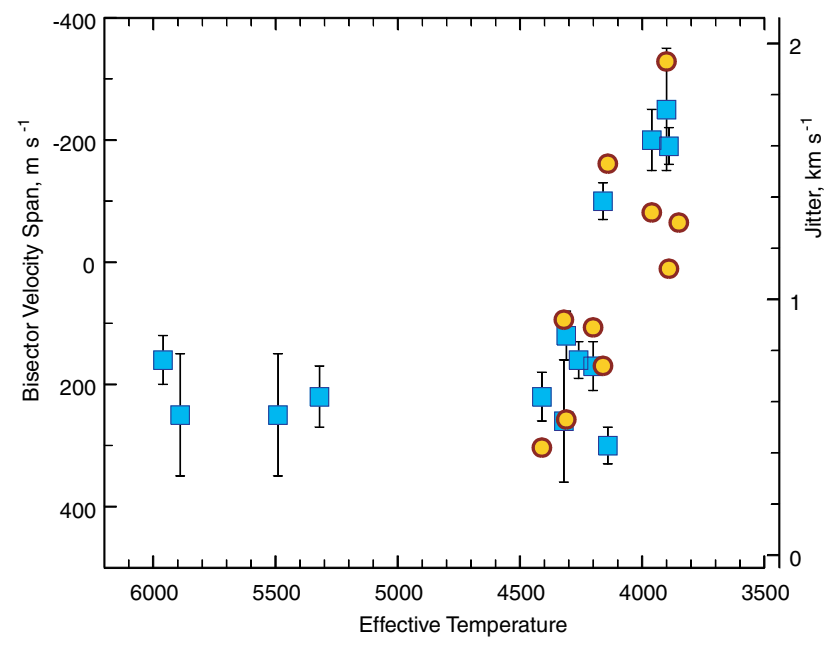

Figure 6. Squares are bisector velocity spans (left ordinate) shown as a function of effective temperature. $\mathrm{C}$ shapes have positive and reversed-C shapes have negative velocity spans. Circles are jitter values (right ordinate).

(A color version of this figure is available in the online journal)

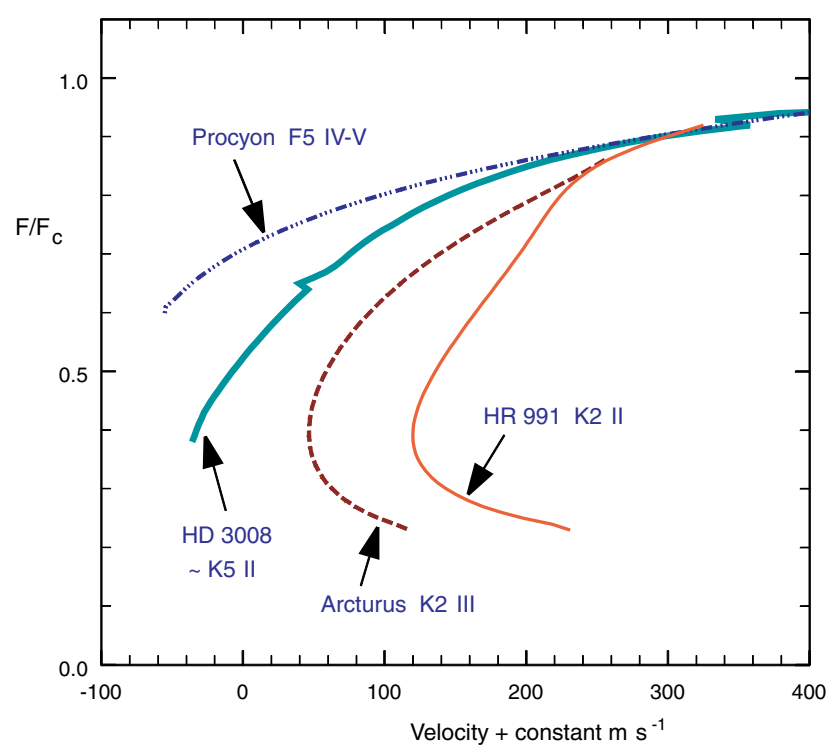

Figure 7. The mean bisector of our program star HD 3308, metallicity $[\mathrm{Fe} / \mathrm{H}]=-1.43$, is compared to bisectors of three disk stars. The $[\mathrm{Fe} / \mathrm{H}]$ value for Procyon is essentially solar; for Arcturus it is near -0.6. HR 991 has roughly the same effective temperature and gravity as HD 3008, but has solar metallicity within the errors of measurement. The three disk-star bisectors are taken from Gray (2005b).

(A color version of this figure is available in the online journal)

more solar-like metallicity. HD 991 is closest to HD 3008 in effective temperature and surface gravity (Luck \& Challener 1995). Although the lines of Procyon and HD 3008 are not as deep as in Arcturus and HD 991, differences in slope in the upper portions of the bisectors are already evident. We conclude that on average, these metal-poor stars show bisector shapes that mimic considerably hotter metal-rich stars.

\section{SUMMARY AND COMMENTS}

Our study confirms the basic granulation signature, the Cshaped bisector, in metal-poor red giant branch stars and the lower luminosity red horizontal-branch stars. However, from our limited sample, it appears that metal-poor stars higher up the red giant branch show reversed-C shapes similar to what is seen for stars on the hot side of the granulation boundary. Additional confirmation is found in a recent study of the supergiant Betelgeuse (M2 Iab, Gray 2008), where the bisectors show the reversed-C shape most of the time (the star is variable). Since Betelgeuse is a Population I star, one might infer that the reversed-C shape is not a result of low metallicity. Rather, the evidence from Betelgeuse suggests that large convection cells and the velocity field within these cells dominate the velocity fields in the photosphere. The rise and fall of the convection cells introduce scatter of a few $\mathrm{km} \mathrm{s}^{-1}$ in radial velocity measurements, the same phenomenon dubbed jitter in earlier studies (e.g., Gunn \& Griffin 1979; Carney et al. 2003).

Clearly we have caught but a glimpse of the dynamical processes going on in red giant branch stars. Just how reversed-C bisectors are connected to velocity jitter is an important question to answer toward understanding large convection cells. One cannot help but wonder how or if reversed-C bisectors of red giant branch stars are related to their counterparts on the hot side of the granulation boundary. Additional high-resolution, high $\mathrm{S} / \mathrm{N}$ observations of these same stars as well as others need to be made. The maverick HD 110281 should be investigated to see if it is an obtuse binary star or if its line profiles show time variation.

It will be interesting to see if hydrodynamical models have as much success in explaining these bisectors as they have with the solar ones (e.g., Asplund et al. 2000).

We are grateful to the Natural Sciences and Engineering Research Council of Canada for financial support. We thank the staff at the Canada-France-Hawaii Telescope for their help and cooperation in acquiring the observations.

\section{REFERENCES}

Allende Prieto, C., García, L., Ramón, J., Lambert, D. L., \& Gustafsson, B. 1999, ApJ, 526, 991

Asplund, M., Nordlund, Å., Trampedach, R., Allende Prieto, C., \& Stein, R. F. 2000, A\&A, 359, 729

Beckers, J. M. 1981, in The Sun as a Star, NASA SP-450, ed. S. Jordan (Washington, DC: CNRS/NASA), 11

Brandt, P. N., \& Solanki, S. K. 1990, A\&A, 231, 221

Carney, B. W., Gray, D. F., Yong, D., Latham, D. W., Manset, N., Zelman, R., \& Laird, J. B. 2008a, AJ, 135, 892

Carney, B. W., Latham, D. W., Stefanik, R. P., Laird, J. B., \& Morse, J. A. 2003, AJ, 125, 293

Carney, B. W., Latham, D. W., Stefanik, R. P., \& Laird, J. B. 2008b, AJ, 135,196

Dravins, D. 1987, A\&A, 172, 200

Dravins, D., Lindegren, L., \& Nordlund, ̊̊ 1981, A\&A, 96, 345

Freytag, B., Steffen, M., \& Dorch, B. 2002, Astron. Nachr., 323, 213

Gray, D. F. 1980, ApJ, 235, 508

Gray, D. F. 1982, ApJ, 255, 200

Gray, D. F. 1983, PASP, 95, 252

Gray, D. F. 1985, PASP, 98,319

Gray, D. F. 1988, Lectures on Spectral-Line Analysis: F, G, \& K Stars (Arva: The Publisher), p 4-39

Gray, D. F. 1989, PASP, 101, 832

Gray, D. F. 2005a, The Observation and Analysis of Stellar Photospheres (3rd ed.: Cambridge: Cambridge Univ. Press), p 443 ff

Gray, D. F. 2005b, PASP, 117, 711

Gray, D. F. 2008, AJ, 135, 1450

Gray, D. F., \& Nagel, T. 1989, ApJ, 341, 421

Gunn, J. E., \& Griffin, R. F. 1979, AJ, 84, 752

Livingston, W. C. 1982, Nature, 297, 208

Luck, R. E., \& Challener, S. L. 1995, AJ, 110, 2968

Schwarzschild, M. 1975, ApJ, 195, 137 